Conclusions We report the largest case series of FMT using frozen stools to treat refractory or recurrent CDI in Hong Kong, showing that it is a safe, efficacious and feasible intervention. Efforts should be made to raise the awareness of this important treatment, with earlier use in difficult-to-treat cases.

\section{IDDF2019-ABS-0192 COMPLIANCE TO PROBIOTIC THERAPY IN IRRITABLE BOWEL SYNDROME IN CLINICAL PRACTICE: A REAL-LIFE STUDY}

${ }^{1}$ Lucrezia Laterza* ${ }^{1}$ Marco Napoli, ${ }^{1}$ Franco Scaldaferri, ${ }^{1}$ Valentina Petito, ${ }^{2}$ Stefano Colombo, 'Antonio Gasbarrini. 'Gastroenterological Area, Gastroenterology And Oncology Department, Fondazione Policlinico Gemelli IRCCS, Catholic University of Rome, Italy; ${ }^{2}$ Beingpharma, Milan, Italy

\subsection{6/gutjnl-2019-IDDFabstracts. 190}

Background Probiotics have been evaluated in multiple clinical trials on irritable bowel syndrome (IBS) showing efficacy on different IBS-related symptoms. Among them, the multistrain probiotic VSL\#3 has been the object in clinical trials evaluating its administration for 4 to 8 weeks. However, in real-life long term compliance could be low. Furthermore, in many country probiotics are fully paid by patients and the cost of therapy could further limit the compliance to probiotic therapy.

Methods This is a single-center, observational, prospective study to evaluate the compliance to prescription of probiotic therapy in real life and to identify factors able to influence adherence to therapy. Patients diagnosed with IBS (either diarrhea, constipation or mix-type) according to Rome IV criteria and receiving a clinical prescription of VSL\#3 for their IBS symptoms were evaluated for eligibility. Patients providing informed consent, were evaluated after 2 months to assess compliance and eventual reasons for discontinuation.

Results Thirty-four patients, twenty-four women (70.6\%) and ten male (29.4\%), have been enrolled. Thirteen of them $(38.2 \%)$ were IBS-D patients, fourteen (41.2\%) were IBS-C patients and six (17.6\%) were IBS-mix patients. The average age was 40.5 years. Twenty-seven of thirty-four patient received a $\underline{4}$ weeks prescription of VSL\#3, whereas five patients received a $\underline{2}$ weeks prescription and two patients an 8 week prescription. All the 34 patients completed the 2 month-follow up for outcomes evaluation. Twenty-one patients $(61.8 \%)$ assumed the therapy as prescribed. Among the thirteen patients (38.2\%) who referred to reduced compliance, nine $(64.3 \%)$ of them assumed at least $50 \%$ of probiotic doses prescribed. The main reasons for discontinuation were low palatability $(n=3)$, bloating $(n=3)$ and price of the product $(n=6)$. One patient enrolled forgot to take the medication. Among AE, bloating has been reported in six (17.6\%) cases. No SAE was reported.

Conclusions According to our results, about 60\% (61.8) of patients assumed all the prescribed probiotic therapy in a real life setting, despite a good safety profile. The main reasons for lack of compliance were palatability, bloating and price of the product.

\section{IDDF2019-ABS-0193 EXPRESSION OF AUTOPHAGY PROTEINS IN THE DUODENUM OF PATIENTS WITH FUNCTIONAL DYSPEPSIA}

Lijun Du*, Binrui Chen, Mengsha Cen. Gastroenterology, Sir Run Run Shaw Hospital, School of Medicine, Zhejiang University, China

\subsection{6/gutjnl-2019-IDDFabstracts. 191}

Background Functional dyspepsia (FD) is considered as an idiopathic disorder with undefined mechanisms. Autophagy plays a role in regulating inflammation, which has been proposed to be associated with the development of FD. In present study, we sought to gain a knowledge on the level of autophagy proteins (beclin1, p62/SQSTM1) in FD patients.

Methods Ten newly diagnosed FD patients and 10 age- and sex-matched asymptomatic controls were consecutively recruited at outpatient gastroenterology clinic of Sir Run Run Shaw Hospital. Endoscopic examination was performed in all participants and mucosa tissues were obtained from the duodenal bulb (D1) and the second portion of the duodenum (D2). Quantitative real-time polymerase chain reaction (qRTPCR) and immunohistochemistry (IHC) were used to evaluate autophagy proteins (beclin1, p62/SQSTM1) at transcriptional and translational levels, respectively.

Results 1. Immunostaining of duodenal tissues revealed a significantly decreased beclin1 protein expression both in D1 $(4.9 \pm 2.7$ vs. $8.0 \pm 3.6, P=0.038)$ and $\mathrm{D} 2(5.7 \pm 2.3$ vs. 9.2 $\pm 2.4, P=0.003$ ) of FD patients compared with asymptomatic controls.

2. When stained for p62, the number of positive cells was significantly higher in D1 $(8.1 \pm 3.2$ vs. $3.7 \pm 2.0, P=0.001)$, but similar in D2 $(10.3 \pm 5.0$ vs. $9.1 \pm 4.3, P=0.661)$ of $\mathrm{FD}$ patients compared with asymptomatic controls.

3. There was no difference of beclin $1 \mathrm{mRNA}$ expression in D1 $(0.01 \pm 0.01$ vs. $0.02 \pm 0.02, P=0.50)$ and D2 $(0.05 \pm 0.03$ vs. $0.07 \pm 0.05, P=0.42$ ) between $\mathrm{FD}$ patients and asymptomatic controls.

4. No difference of p62 mRNA expression in D1 $(0.03$ \pm 0.02 vs. $0.05 \pm 0.04, P=0.22)$ and $\mathrm{D} 2(0.02 \pm 0.01$ vs. 0.03 $\pm 0.03, P=0.33$ ) was observed between FD patients and asymptomatic controls.

Conclusions The findings of this study provided that autophagy protein beclin 1 was down-regulated in the duodenum and p62 was up-regulated in D1 of patients with FD, which indicated abnormal autophagy is potentially related to FD development.

\section{IDDF2019-ABS-0195 EFFICACY AND SAFETY OF ENDOSCOPIC ULTRASOUND-GUIDED BILIARY DRAINAGE IN PATIENTS OF FAILED ERCP}

Chun-Tao Liu*, Peng Li, Shu-Tian Zhang. Department of Gastroenterology, Beijing Friendship Hospital, Capital Medical University, China

\subsection{6/gutjn|-2019-IDDFabstracts. 192}

Background Endoscopic retrograde cholangiography (ERCP) is widely used as a first-line intervention for biliary obstruction. 